\title{
Alopecia androgénica femenina. Nuevas herramientas terapéuticas frente a los factores fisiopatológicos implicados: hormonal, oxidativo e inflamatorio
}

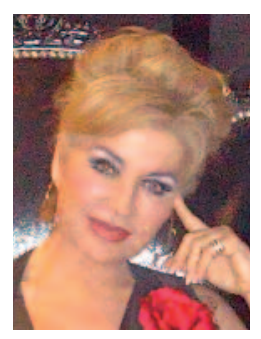

\section{Aurora Guerra Tapia}

Jefa de Sección de Dermatología. Hospital Universitario 12 de Octubre. Madrid. Profesora titular de Dermatología. Universidad Complutense de Madrid.

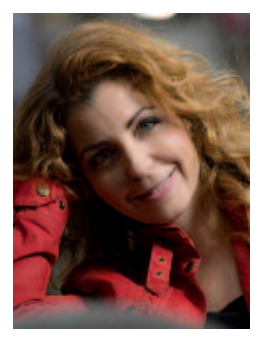

\section{Elena González-Guerra} Doctora en Medicina y Cirugía. Médico adjunto del Servicio de Dermatología. Hospital Clínico San Carlos. Madrid. Profesora asociada de Dermatología. Facultad de Medicina. Universidad Complutense de Madrid.

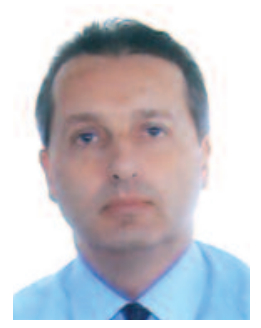

José M.a Borrás Schierloh Licenciado en Farmacia. Licenciado en Medicina y Cirugía. Investigación y Desarrollo. Laboratorios Reig Jofre. Barcelona.

\section{INTRODUCCIÓN}

Actualmente, se considera que existen otros factores, además del hormonal, implicados de una manera importante en la fisiopatología de la alopecia androgénica femenina (AAF), tales como el estrés oxidativo y la microinflamación ${ }^{1}$. Un análisis en profundidad de estos factores justifica nuevas propuestas de tratamiento con una visión terapéutica más amplia, que mejorarán la eficacia en el tratamiento de la AAF.

A lo largo de este artículo, se realizarán dos planteamientos como propuestas de futuro para lograr un abordaje más integral en el tratamiento de la AAF; por un lado, se planteará conseguir un incremento de la actividad inhibidora de la enzima $5 \alpha$-reductasa con los extractos orales actualmente disponibles que han mostrado mejor eficacia y seguridad en el tratamiento de la AAF (factor hormonal) y, por otro lado, plantearemos contemplar el uso de una nueva molécula, también oral, para el tratamiento de la AAF capaz de ofrecer una herramienta terapéutica más frente a los nuevos factores fisiopatológicos identificados en la patogenia de la AAF (estrés oxidativo, microinflamación), molécula capaz de actuar de manera sinérgica con los extractos anteriores, aportando efectos beneficiosos sobre los elementos esenciales del ciclo de vida del cabello.

Mostraremos las bases de estos planteamientos, realizando un análisis de la evidencia científica disponible, y expondremos los resultados obtenidos en estudios in vitro, que dan el soporte suficiente para apoyar la práctica de las propuestas desde el punto de vista clínico. 
Entre las alternativas de tratamiento actualmente disponibles con alta actividad inhibidora de la enzima $5 \alpha$-reductasa, se encuentran los extractos lipídicos de Serenoa repens y Pygeum africanum ${ }^{2,3}$, que han mostrado ser una elección terapéutica eficaz y bien tolerada. Apoyados en esta certeza, plantearemos incrementar su actividad inhibidora aumentando su dosis diaria, manteniendo su buena tolerabilidad e incrementando su efectividad en el tratamiento.

En cuanto a los nuevos factores oxidativo e inflamatorio implicados en la fisiopatología de la AAF, plantearemos la incorporación de un nuevo ingrediente activo con efectos antioxidante y antiinflamatorio que se muestre capaz de incrementar la eficacia del tratamiento: metilsulfonilmetano (MSM).

El MSM es una fuente de azufre orgánico esencial para la síntesis de aminoácidos azufrados, colágeno y queratina, esenciales para el ciclo vital del cabello, que, además, tiene propiedades antiinflamatorias, antioxidantes y antirradicales libres ${ }^{1,4}$, y que ya ha mostrado resultados positivos en el tratamiento de problemas capilares. Mostraremos los resultados obtenidos en estudios diseñados con células de folículo piloso, para objetivar el efecto antioxidante y antiinflamatorio que la incorporación del MSM podría ofrecer para mejorar la eficacia en el tratamiento de la AAF.

\section{FACTOR HORMONAL EN LA ALOPECIA ANDROGÉNICA FEMENINA}

\section{Alternativas disponibles para inhibir la enzima $\mathbf{5} \alpha$-reductasa}

Desde hace años, conocemos el papel que desempeñan los inhibidores de la $5 \alpha$-reductasa en el tratamiento de la hiperplasia benigna de próstata (HBP) y de la AAF. Los tratamientos usados en estas enfermedades son los inhibidores sintéticos de la $5 \alpha$-reductasa (finasterida y dutasterida) en los varones, así como antiandrógenos específicos (acetato de ciproterona o acetato de ciproterona + etinilestradiol) en las mujeres. Sin embargo, el uso de todos ellos está contraindicado en mujeres durante el embarazo y la lactancia, y tienen una serie de efectos adversos que a menudo impiden su uso en el tratamiento de la $\mathrm{AAF}^{5,6}$.

Frente a este panorama terapéutico, se estudió un grupo de sustancias de origen natural, con propiedades inhibidoras de la $5 \alpha$-reductasa y de las que se tenía experiencia clínica por su empleo en el tratamiento de la HBP y para el tratamiento en mujeres de trastornos urológicos ocasionados por congestión pélvica, como son los extractos lipídicos de Serenoa repens y de Pygeum africanum ${ }^{2,3}$, destacando su buen perfil de seguridad y su carencia de efectos negativos sobre la libido y la esfera sexual de la mujer.

La revisión Cochrane publicada en 2000 acerca de la eficacia de los extractos vegetales inhibidores de la $5 \alpha$-reductasa en la $\mathrm{HBP}^{7}$ concluyó que Serenoa repens es el extracto que presenta más evidencia científica sobre su actividad inhibidora de la $5 \alpha$-reductasa y, en segundo lugar, Pygeum africanum, pudiéndose asociar para incrementar sus efectos, actuando de manera sinérgica en la inhibición de la enzima $5 \alpha$-reductasa.

La misma revisión sitúa en un escalón inferior de evidencia científica los extractos de Hypoxis rooperi y Secale cereale y, en las últimas posiciones, los extractos de Urtica dioica y Cucurbita pepo (semilla de calabaza) por este orden. Más concretamente, dicha revisión dice textualmente que «no hay evidencia convincente que respalde el uso de Urtica dioica y Cucurbita pepo en solitario", aconsejando su utilización en asociación con alguno de los que tienen evidencia más contrastada (por ejemplo, Serenoa repens o Pygeum africanum).

En cuanto la experiencia clínica con la combinación de los extractos Serenoa repens y Pygeum africanum, se publicó en 2016 un ensayo clínico comparativo del producto RJ-SP4ACA (marca comercial Complidermol $5 \alpha$ ) frente a placebo, realizado en 40 mujeres con un promedio de edad de 58 años diagnosticadas de AAF de nivel I-II según la escala de Ludwig ${ }^{8}$. Tras 16 semanas de tratamiento, 
se observó un aumento estadísticamente significativo (nivel de significación $[p]<0,05$ ) de un 12,32\% en la cantidad de cabellos en fase anágena, así como un descenso casi equivalente $(12,25 \%)$ de cabellos en fase telógena, y un aumento del $50 \%$ en la resistencia del cabello a la tracción $(p<0,05)$. De este modo, se observó reversión del cabello a un estado casi similar al de un cabello sano, libre de AAF (fig. 1).

Por otro lado, y respecto al perfil de seguridad observado en la investigación, tanto el tratamiento activo como el placebo fueron seguros y bien tolerados durante todo el estudio. La frecuencia de efectos adversos fue baja y la mayoría de carác- ter leve y transitorio (únicamente tres pacientes del grupo activo refirieron la sensación de molestias gastrointestinales muy leves durante el estudio). Tal es así, que, si analizáramos la frecuencia de los efectos adversos notificados, tanto de manera espontánea por los profesionales de la salud como en las búsquedas bibliográficas sistemáticas a lo largo de los años de comercialización del suplemento alimenticio objeto del estudio anterior, encontraríamos que se ha registrado un solo caso de efecto adverso leve por cada 176000 tratamientos a lo largo de 10 años de su comercialización.

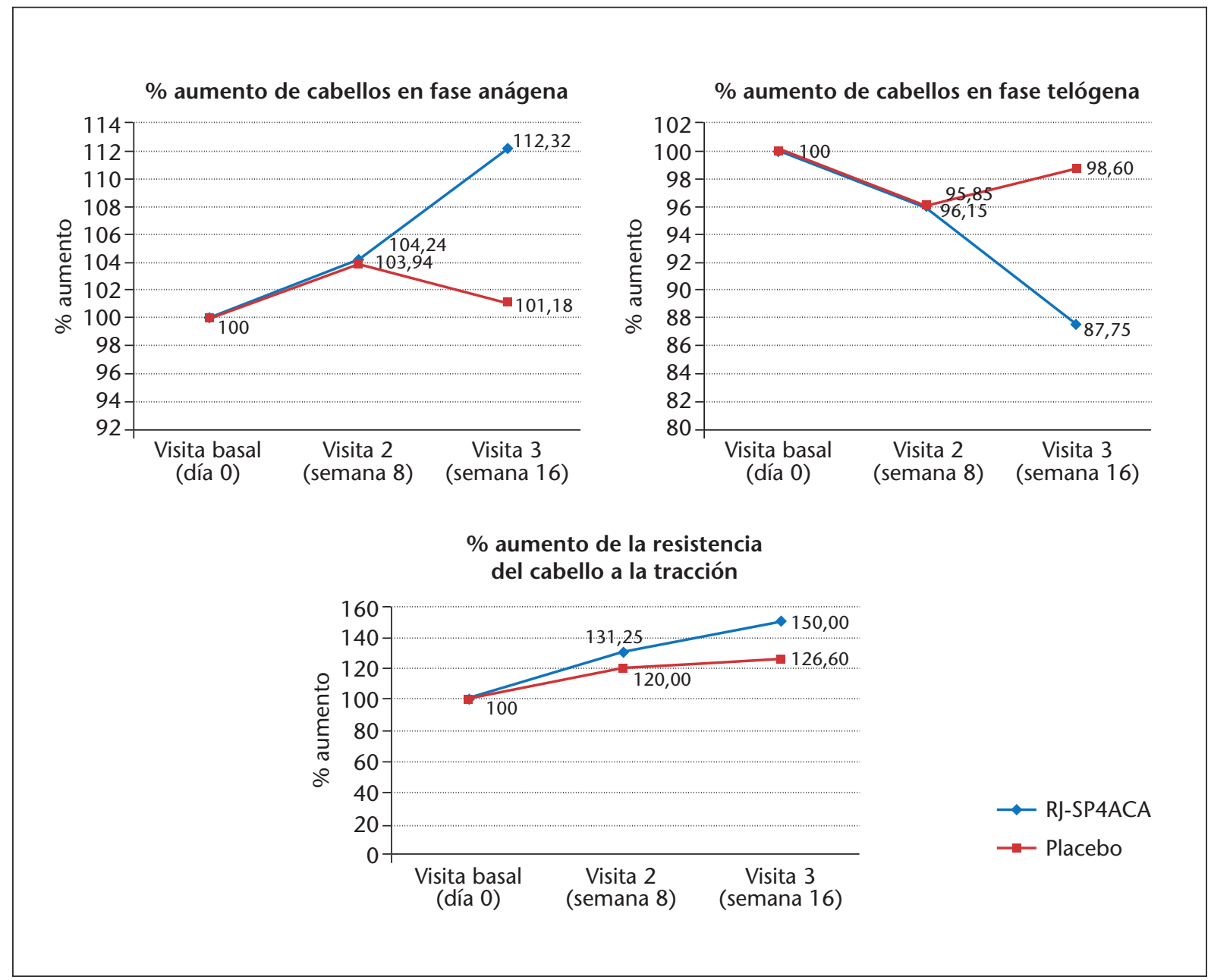

Figura 1. Evolución de los cabellos en fase anágena y telógena, y resistencia a la tracción a las 16 semanas de tratamiento ${ }^{8}$. 
Alopecia androgénica femenina. Nuevas herramientas terapéuticas frente a los factores fisiopatológicos implicados: hormonal, oxidativo e inflamatorio

\section{Estudio del incremento de la actividad inhibidora de la enzima $5 \alpha$-reductasa}

Una vez identificados los extractos lipídicos de Serenoa repens y de Pygeum africanum como los de mayor soporte científico en cuanto a su actividad inhibidora de la enzima $5 \alpha$-reductasa en el tratamiento de la $\mathrm{AAF}$, podría plantearse un incremento de esta actividad suponiendo este efecto dependiente de la dosis y teniendo en cuenta que el margen de tolerabilidad de estos ingredientes activos es muy amplio.

Los extractos lipídicos de Pygeum africanum y Serenoa repens tienen avalada su seguridad por los años de utilización en la HBP con dosis que van desde los 100-200 mg/día para Pygeum hasta los $300 \mathrm{mg} /$ día para Serenoa sin que se detecten efectos secundarios remarcables. La Organización Mundial de la Salud, en su monografía WHO monographs on selected medicinal plants - Volume $2^{9}$, describe que los extractos de Pygeum y Serenoa son bien tolerados en humanos en las dosis recomendadas de uso.
En este sentido, lo primero que cabría preguntarse es en qué medida el efecto que encontraremos al incrementar la dosis es directamente proporcional al aumento de esta. Para determinar este valor incremental, se ha realizado un estudio in vitro comparativo sobre la capacidad de inhibición de la $5 \alpha$-reductasa en función de la cantidad de extracto puro de Serenoa y Pygeum asociado, en tres concentraciones diferentes: $20 \mu \mathrm{g} / \mathrm{ml}, 100 \mu \mathrm{g} / \mathrm{ml}$ y $400 \mu \mathrm{g} / \mathrm{ml}$. Los resultados obtenidos ponen de manifiesto que existe una relación proporcional entre la cantidad de extracto de Pygeum + Serenoa y su actividad inhibidora de la $5 \alpha$-reductasa.

Como se puede ver en las siguientes gráficas, con el aumento de la cantidad de extracto de Pygeum + Serenoa, se observa un incremento de la acción inhibidora de los extractos sobre la enzima $5 \alpha$-reductasa (fig. 2).

Como conclusión a este primer planteamiento, existe margen de eficacia y seguridad suficiente como para proponer un incremento de dosis de los extractos Pygeum + Serenoa, que cuen-

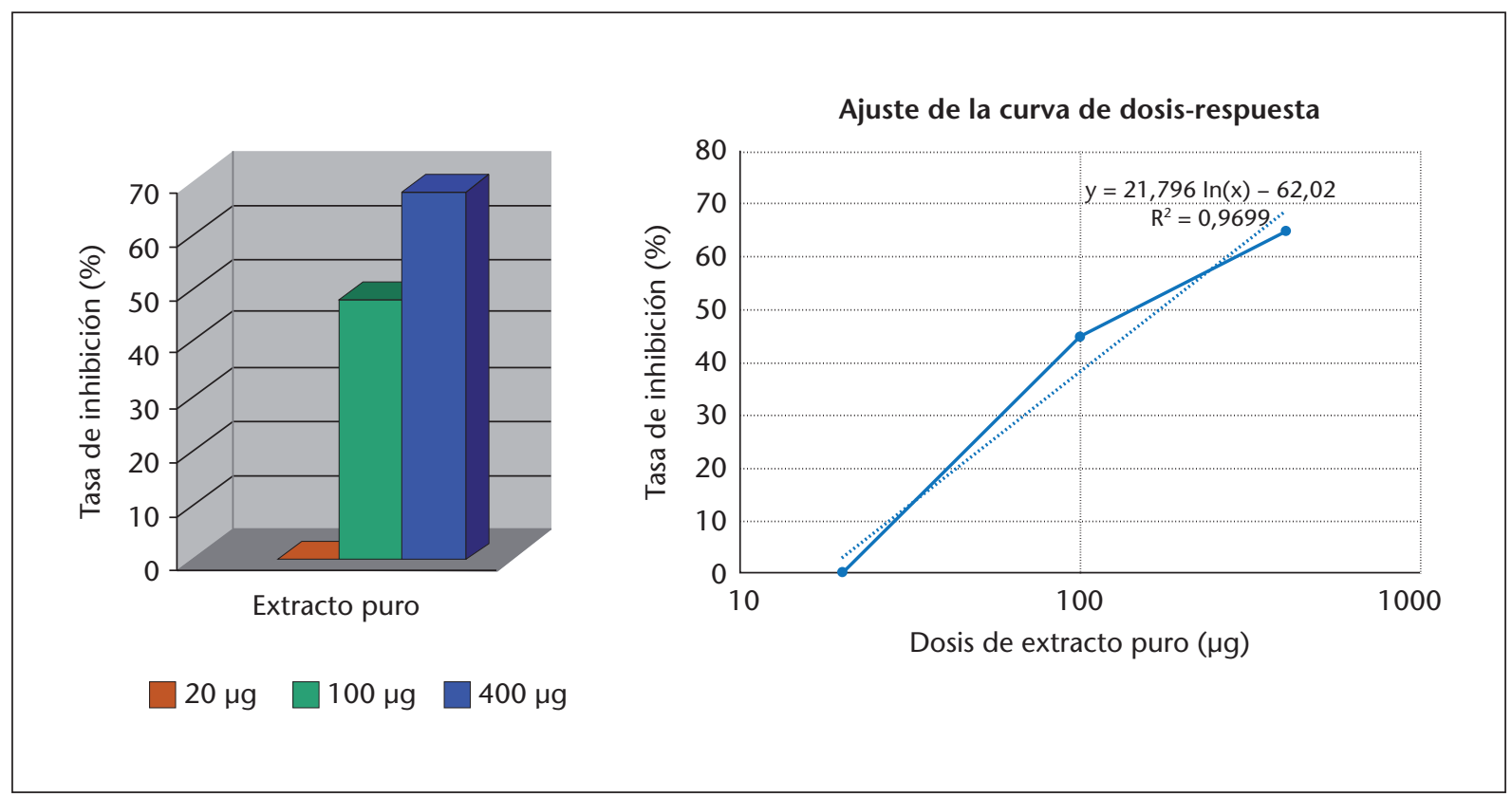

Figura 2. Efectos inhibidores en distintas dosis de extracto de Pygeum + Serenoa sobre la $5 \alpha$-reductasa in vitro. 
tan con el aval suficiente de evidencia científica publicada, y conseguir un incremento de su actividad inhibidora de la enzima $5 \alpha$-reductasa. Recordemos que conseguir esta inhibición es el primer objetivo en el tratamiento de la AAF, es decir, el tratamiento del factor hormonal con la inhibición de la enzima $5 \alpha$-reductasa que reduce el paso de testosterona a dihidrotestosterona o DHT (andrógeno cinco veces más afín a los receptores androgénicos que la testosterona), responsable mayoritario de la miniaturización de los folículos pilosos por un mecanismo de apoptosis celular.

\section{FACTOR OXIDATIVO E INFLAMATORIO EN LA ALOPECIA ANDROGÉNICA FEMENINA}

\section{Análisis del metilsulfonilmetano como agente con efecto antioxidante y antiinflamatorio en la alopecia androgénica femenina}

\section{Origen y síntesis del metilsulfonilmetano}

El MSM es una molécula pequeña y de bajo peso molecular $(94,13 \mathrm{~g} / \mathrm{mol})$ que forma parte en la naturaleza del llamado «ciclo del azufre» ${ }^{10}$. El ciclo del azufre se inicia en las algas y el plancton marino, microorganismos capaces de absorber considerables cantidades de azufre inorgánico procedente del agua de mar y convertirlo en moléculas elementales de azufre orgánico. Cuando estos microorganismos mueren, las moléculas de azufre son transformadas en dimetilsulfuro, un compuesto volátil poco soluble en el agua de mar y que se evapora fácilmente. Una vez en la atmósfera, los rayos ultravioleta (UV) del sol lo oxidan a dimetilsulfóxido (DMSO) y, posteriormente, a MSM. Ambos compuestos son muy solubles en agua y se concentran en el vapor atmosférico, retornando de nuevo a la tierra disueltos en la lluvia ${ }^{10}$. Una vez de nuevo en la tierra, el MSM es absorbido por las plantas, los árboles frutales, etc., pasando a formar parte de la alimentación de humanos y animales. Podemos encontrar concentraciones de $1-4 \mathrm{mg} / \mathrm{kg}$ de MSM en vegetales y frutas, y concentraciones de $2-5 \mathrm{mg} / \mathrm{l}$ en la leche de las vacas que se alimentan de hierba fresca ${ }^{10}$.

Así pues, el MSM es una fuente de azufre elemental, que forma parte de nuestra dieta habitual, y que tiene la ventaja de ser muy fácilmente absorbible por su pequeño tamaño, por lo que no causa trastornos gástricos y, a igualdad de dosis, aporta mayor cantidad de azufre que los aminoácidos azufrados, tales como la cistina ${ }^{8}$. Tal es así que $500 \mathrm{mg}$ de MSM aportan la misma cantidad de azufre que $640 \mathrm{mg}$ de L-cistina.

Antecedentes del uso de metilsulfonilmetano La utilización terapéutica del MSM se inicia gracias a su capacidad de fomentar la síntesis de sustancias azufradas que se encuentran en el organismo humano, principalmente, colágeno y queratina. Por su facultad de incrementar esta síntesis de colágeno, empezó a utilizarse en procesos degenerativos e inflamatorios de las articulaciones ${ }^{11}$. De su utilización en dichas enfermedades, pudo constatarse que no solamente intervenía en la síntesis del colágeno articular, sino que todas las estructuras formadas por tejido conectivo mejoraban significativamente con la ingesta de una dosis diaria mínima de $1 \mathrm{~g}$ de MSM. Concretamente, se observaron mejoras significativas en el estado de la piel y las faneras, hasta el punto de ser denominado «beauty mineral ${ }^{11}$. De esta manera, no solo se pudo comprobar su efecto beneficioso en procesos degenerativos e inflamatorios de las articulaciones, sino que se pudieron constatar las siguientes tres acciones, muy útiles para contrarrestar los procesos dermatológicos ligados a la etiopatogenia de la $\mathrm{AAF}^{12}$ :

- Incremento de la síntesis de queratina.

- Actividad antioxidante: impide la propagación de especies reactivas del oxígeno (ERO).

- Actividad antiinflamatoria: reduce la presencia de factores inflamatorios. 
Gracias a todas estas observaciones, uno de los principales campos de uso del MSM como producto comercializado se desarrolla actualmente en el tratamiento del cabello y de las uñas. Buen ejemplo de esta afirmación es que el MSM en los Estados Unidos lleva años formando parte de la composición de productos para el tratamiento de problemas capilares, ya que es capaz de desarrollar una triple acción, que otros aminoácidos azufrados, como la cistina, no reali$\operatorname{zan}^{12}$.

Así pues, aparte de ser una fuente ideal de azufre biológico para la síntesis de colágeno y queratina, las otras dos propiedades del MSM (antioxidante y antiinflamatorio), que ya se mostraron muy útiles en el tratamiento de procesos degenerativos de las articulaciones, resultan muy interesantes para tratar los nuevos factores fisiopatológicos identificados en la $\mathrm{AAF}^{12}$.

\section{Incorporación del metilsulfonilmetano con efecto antioxidante y antiinflamatorio}

\section{El metilsulfonilmetano como agente antioxidante en el folículo piloso}

Durante el estrés oxidativo, tienden a aumentar los niveles plasmáticos de los marcadores de oxidación, como el malondialdehído (MDA) —indicador de peroxidación lipídica-, la mieloperoxidasa (MPO) -indicativa de la presencia de ERO- y las proteínas carboniladas (PC) - indicadoras de oxidación proteica- - Al mismo tiempo, los niveles de antioxidantes, como el glutatión (GSH), la catalasa (CAT) o la superóxido-dismutasa (SOD), tienden a reducirse por el consumo que se produce por parte de las $\mathrm{ERO}^{13}$.

El desequilibrio entre los factores antioxidantes y oxidantes a favor de un exceso de estrés oxidativo ha sido identificado como uno de los factores implicados en la AAF, como un potente inhibidor del crecimiento del cabello, y se le ha vinculado a un aumento de la apoptosis de las células de las papilas del folículo pilosebáceo en las zonas alopécicas, y a una menor capacidad de proliferación de estas células ${ }^{1}$.

Respecto al equilibrio descrito anteriormente entre agentes antioxidantes y oxidantes, el MSM ha demostrado poseer propiedades antioxidantes, siendo capaz de mantener $-\mathrm{o}$, incluso, de aumentar- los niveles de factores antioxidantes como el GSH, la CAT o la SOD y, al mismo tiempo, impedir el incremento de los factores oxidantes como el MDA, la MPO o las $\mathrm{PC}^{13}$.

Estos efectos del MSM se han podido constatar en estudios llevados a cabo con deportistas a los que se les sometía a un ejercicio físico violento provocador de estrés oxidativo y se les administraba un suplemento con MSM antes del ejercicio. Se observó que el MSM fue capaz de incrementar los niveles de GSH y reducir los de glutatión oxidado (GSSG), al mismo tiempo que disminuyó los incrementos de MDA (indicador de peroxidación lipídica) y de PC (indicadoras de oxidación proteica) ${ }^{13}$.

Con el fin de demostrar estos mismos efectos antioxidantes del MSM en el folículo piloso, se han llevado a cabo estudios en la papila dérmica del folículo humano (HFDPC) in vitro, con diferentes diluciones de MSM que permitían mantener estable el medio de cultivo celular y, a la vez, medir el efecto del MSM sobre la expresión génica de los siguientes factores antioxidantes: la SOD-1, la CAT y el MDA, indicador de peroxidación lipídica.

Las HFDPC fueron cultivadas en presencia de tres concentraciones diferentes $(0,05 \mathrm{mg} / \mathrm{ml}$, $0,1 \mathrm{mg} / \mathrm{ml} \mathrm{y} 0,5 \mathrm{mg} / \mathrm{ml}$ ) de MSM durante 24 horas. Tanto al grupo de control de cultivo celular sin MSM como a los cultivos con MSM se les sometió a un estrés oxidativo consistente en la exposición a rayos ultravioleta $B$ (UVB). Después del tratamiento, el ácido ribonucleico (ARN) total se purificó, se cuantificó y se utilizó para sintetizar ácido desoxirribonucleico complementario (ADNc). Este ADNc de células tratadas o no tratadas (control) se usó para determinar la expresión génica relativa de SOD-1, CAT y MDA, mediante reac- 
ción en cadena de la polimerasa (PCR) cuantitativa en tiempo real. Se usó actina como gen de referencia para determinar la cantidad relativa de ARN mensajero en todas las muestras.

Se obtuvieron los siguientes resultados:

\section{Efecto del metilsulfonilmetano sobre} la expresión de la superóxido-dismutasa 1

Los resultados mostraron que el tratamiento con MSM en dosis de $0,05 \mathrm{mg} / \mathrm{ml}$ tuvo un efecto incremental del 13,5 $\pm 10,6 \%$ sobre la expresión de SOD-1, respecto del grupo de control sin MSM (fig. 3).

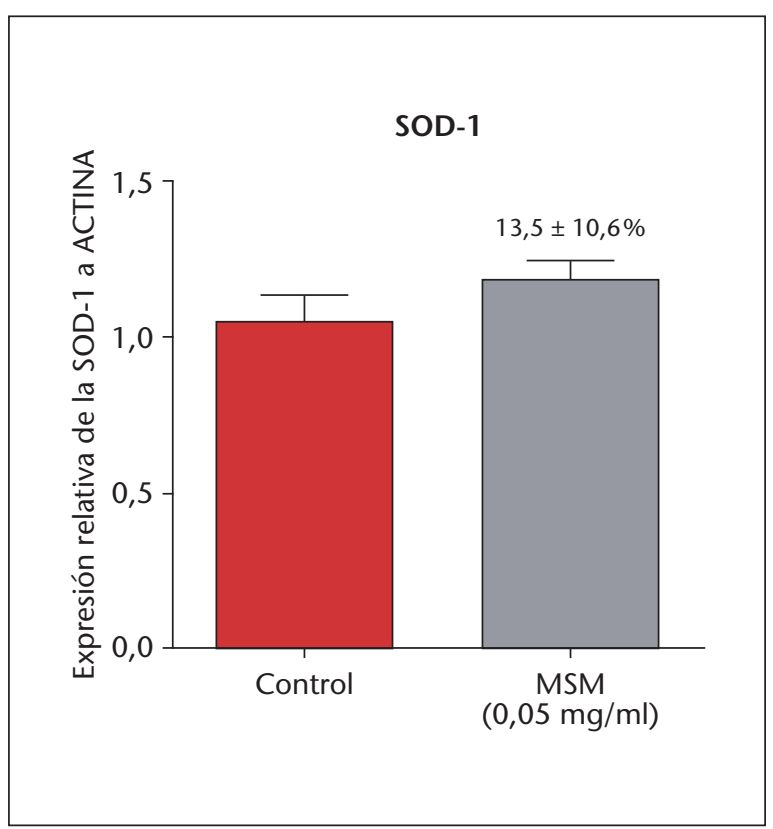

Figura 3. Efecto del metilsulfonilmetano (MSM) sobre la expresión de la superóxido-dismutasa 1 (SOD-1).

\section{Efecto del metilsulfonilmetano sobre la expresión de la catalasa}

Los resultados mostraron que el tratamiento con MSM en dosis de $0,5 \mathrm{mg} / \mathrm{ml}$ tuvo un efecto incremental significativo del 13,1 $\pm 2,9 \%$ sobre la expresión de CAT, respecto del grupo de control sin MSM (fig. 4).

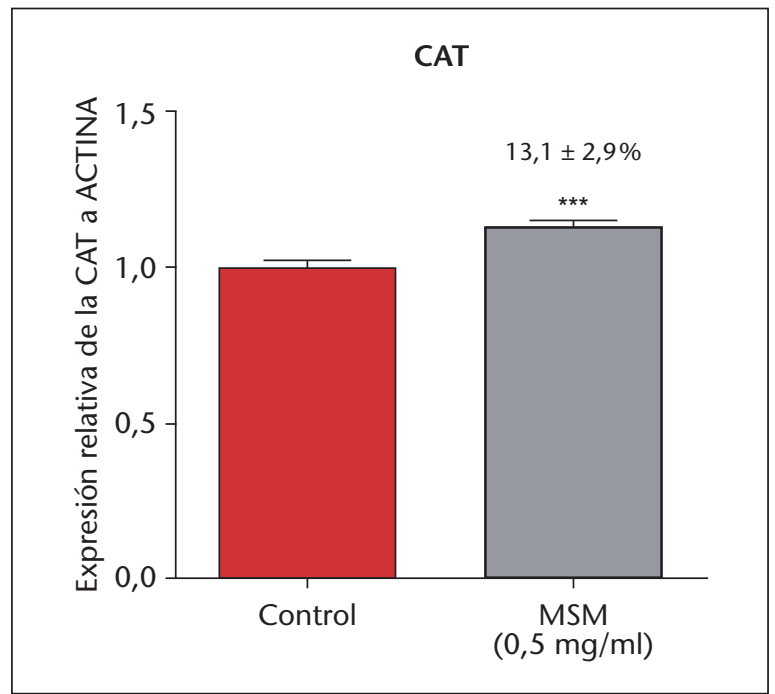

Figura 4. Efecto del metilsulfonilmetano (MSM) sobre la expresión de la catalasa (CAT).

\section{Efecto del metilsulfonilmetano sobre la expresión del malondialdehído}

Los resultados mostraron que el tratamiento con MSM en dosis de $0,5 \mathrm{mg} / \mathrm{ml}$ tuvo un efecto de reducción significativa del $75,4 \pm 13,9 \%$ sobre la expresión del MDA, respecto del grupo de control sin MSM (fig. 5).

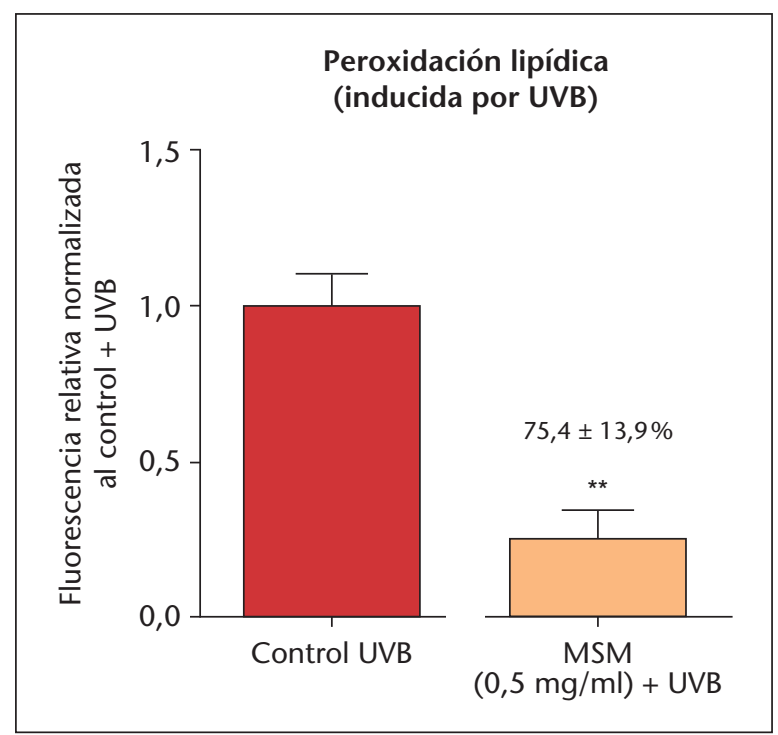

Figura 5. Efecto del metilsulfonilmetano (MSM) sobre la expresión de malondialdehído (MDA).

UVB: radiación ultravioleta $B$. 
De acuerdo con los datos de este estudio, podemos concluir que el tratamiento con MSM aplicado en la HFDPC mostró efectos significativos de incremento de la expresión de los factores antioxidantes SOD-1 y CAT, y redujo de manera significativa la expresión del MDA, siendo este el principal factor indicador de la peroxidación lipídica.

Con los datos observados in vitro sobre los efectos del MSM en la HFDPC, se podría plantear el uso de MSM en el tratamiento de la AAF, en busca de sus efectos beneficiosos antioxidantes, como tratamiento del factor oxidativo identificado como uno de los factores implicados en la AAF y responsable del acortamiento del ciclo de vida del cabello con el efecto de aceleración del paso de la fase anágena a la fase catágena, así como uno de los responsables de la miniaturización de los folículos pilosos por favorecer el mecanismo de apoptosis sobre las células de la papila dérmica.

\section{El metilsulfonilmetano como agente antiinflamatorio en el folículo piloso}

La respuesta de los queratinocitos al estrés oxidativo, irritantes, radiación UV, etc. es la liberación intracelular de la interleucina 1 alfa (IL-1 $\alpha$ ) almacenada. En respuesta a dicha liberación, se inicia la transcripción de otros genes sensibles de los queratinocitos adyacentes, y se liberan otros intermediarios inflamatorios como la interleucina 1 beta (IL-1 $\beta$ ), el factor de necrosis tumoral alfa (TNF- $\alpha$ ), el factor de crecimiento transformante beta 1 (TGF- $\beta 1$ ) y la interleucina 6 (IL-6), entre otros. Estas sustancias acaban estimulando a neutrófilos, macrófagos y fibroblastos, que contribuyen a perpetuar el mecanismo fisiológico de la inflamación ${ }^{1}$.

Este fenómeno de microinflamación en las zonas del cuero cabelludo con AAF, con infiltración de linfocitos $\mathrm{T}$ y macrófagos en el tercio superior del folículo piloso cerca del infundíbulo, es lo que se ha identificado como factor implicado en la AAF responsable de originar cambios tisulares y producir fibrosis perifolicular en la alopecia androgénica, que daría lugar a un agrandamiento de la glándula sebácea, un engrosamiento de la vaina dérmica y un depósito anómalo de fibras de colágeno en la parte inferior del folículo piloso. La presencia de microinflamación en el tercio superior del folículo y la fibrosis contribuyen al proceso de miniaturización del cabello y a la apoptosis celular observada en la $\mathrm{AAF}^{1}$.

Con el objeto de estudiar los efectos antiinflamatorios del MSM en el folículo piloso, se ha desarrollado un estudio in vitro que ha buscado evaluar los efectos antiinflamatorios del MSM en dosis de $0,05 \mathrm{mg} / \mathrm{ml}$ sobre la HFDPC, observando su efecto en diversos factores proinflamatorios, específicamente, el TNF- $\alpha$, la IL-1 $\alpha$, la IL-6, la interleucina 8 (IL-8) y el TGF- $\beta$.

Con el fin de inducir un estado inflamatorio en las células, estas fueron tratadas con LPS (lipopolisacárido), una molécula grande que consta de un lípido y un polisacárido, presente en la membrana externa de las bacterias gramnegativas y que es capaz de provocar un incremento de la respuesta inmunitaria en los animales.

\section{Efecto sobre la expresión del factor de necrosis tumoral alfa}

Los resultados mostraron que el tratamiento con MSM en dosis de $0,05 \mathrm{mg} / \mathrm{ml}$ tuvo un efecto de reducción significativa del TNF- $\alpha$ en un $45,1 \pm$ 29,9\%, en comparación con el control + LPS, resultando un descenso significativo $(p<0,0001)$ (fig. 6).

\section{Efecto sobre la expresión de la interleucina 1 alfa}

Los resultados mostraron que el tratamiento con MSM en dosis de $0,05 \mathrm{mg} / \mathrm{ml}$ tuvo un efecto de reducción significativa de la IL-1 $\alpha$ en un $49,9 \pm 29,1 \%$, en comparación con el control + LPS, resultando un descenso significativo $(p<0,0001)$ (fig. 7).

\section{Efecto sobre la expresión de la interleucina 6}

Los resultados mostraron que el tratamiento con MSM en dosis de $0,05 \mathrm{mg} / \mathrm{ml}$ tuvo un efecto de reducción significativa de la IL-6 en un 
TNF- $\alpha$ (LPS)

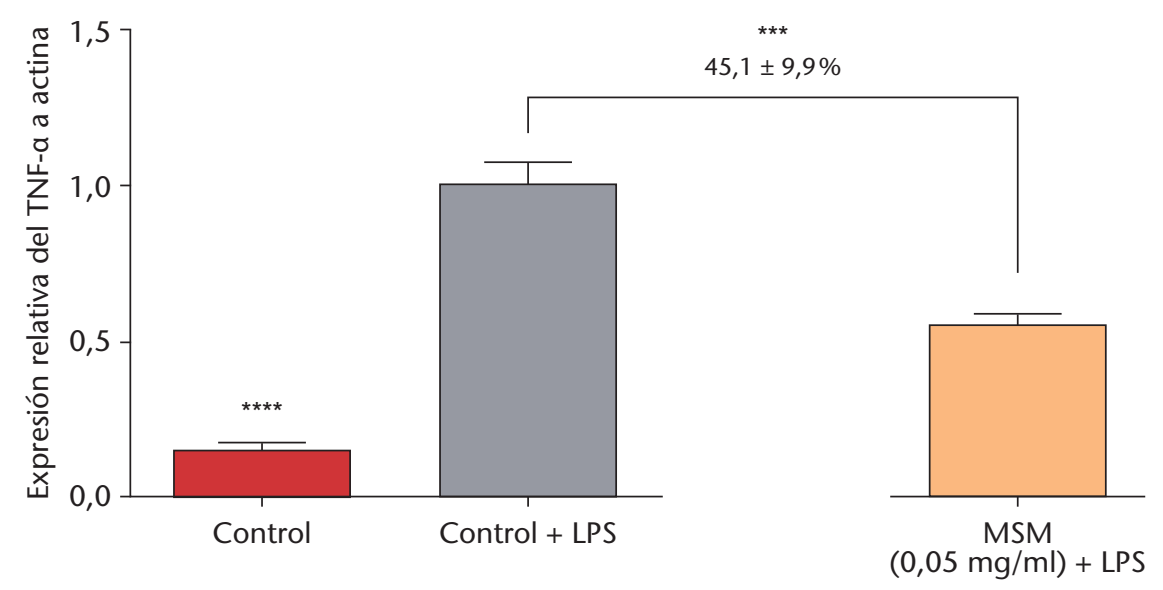

Figura 6. Efecto del metilsulfonilmetano (MSM) sobre la expresión del factor de necrosis tumoral alfa (TNF- $\alpha$ ). LPS: lipopolisacárido.

IL-1 $\alpha$ (LPS)

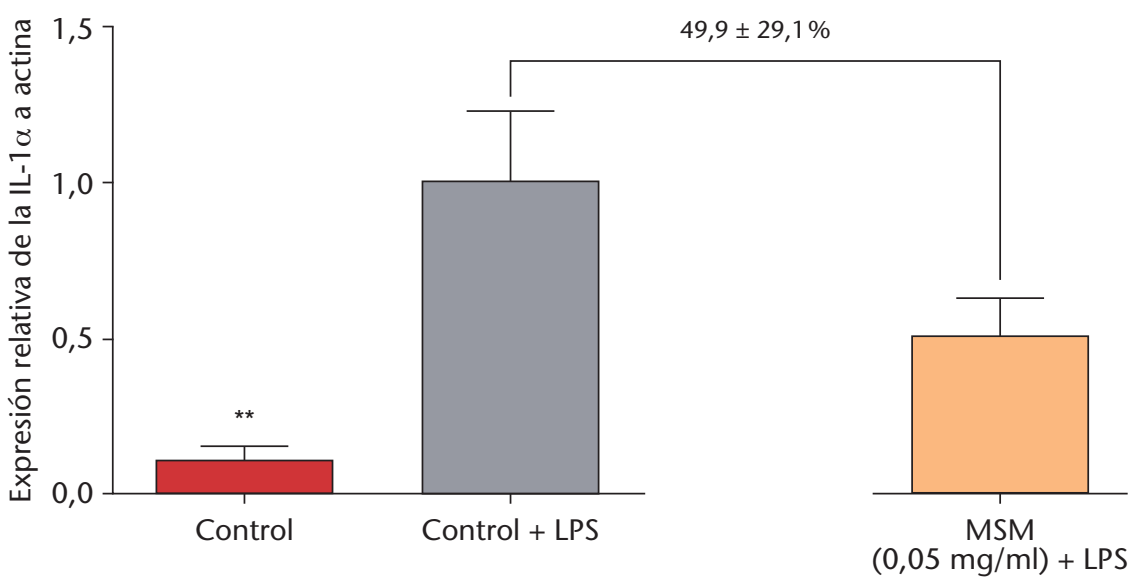

Figura 7. Efecto del metilsulfonilmetano (MSM) sobre la expresión de la interleucina 1 alfa (IL-1 $\alpha$ ).

LPS: lipopolisacárido.

73,3 $\pm 29,9 \%$, en comparación con el control

+ LPS, resultando un descenso significativo $(p<0,0001)$ (fig. 8).
Efecto sobre la expresión de la interleucina 8

Los resultados mostraron que el tratamiento con MSM en dosis de $0,05 \mathrm{mg} / \mathrm{ml}$ tuvo un efecto de 
Alopecia androgénica femenina. Nuevas herramientas terapéuticas frente a los factores fisiopatológicos implicados: hormonal, oxidativo e inflamatorio

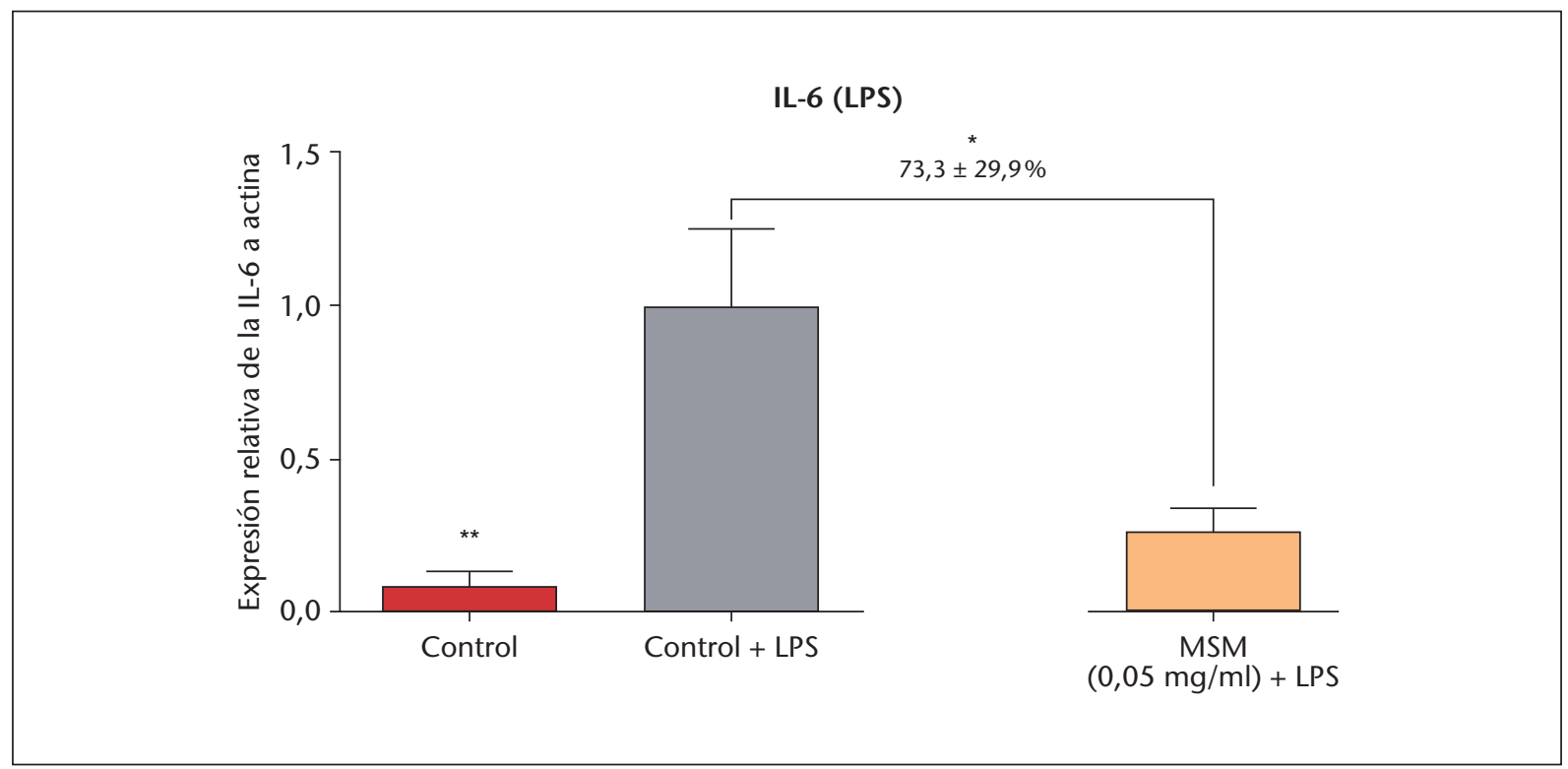

Figura 8. Efecto del metilsulfonilmetano (MSM) sobre la expresión de la interleucina 6 (IL-6).

LPS: lipopolisacárido.

reducción significativa de la IL-8 en un 63,1 \pm $30,4 \%$, en comparación con el control + LPS, resultando un descenso significativo $(p<0,0001)$ (fig. 9).
Efecto sobre la expresión del factor de crecimiento transformante beta

Los resultados mostraron que el tratamiento con MSM en dosis de $0,05 \mathrm{mg} / \mathrm{ml}$ tuvo un efecto de

\section{IL-8 (LPS)}

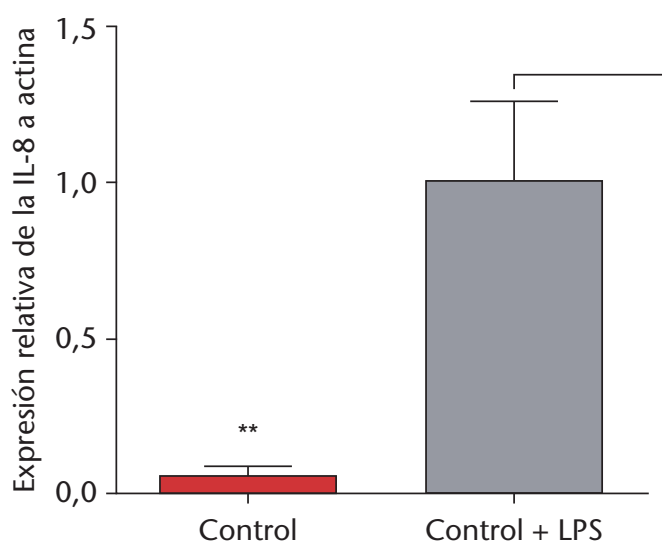

$63,1 \pm 30,4 \%$

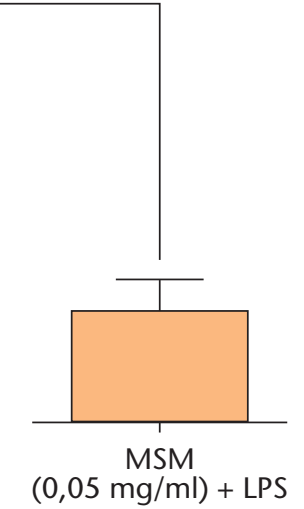

Figura 9. Efecto del metilsulfonilmetano (MSM) sobre la expresión de la interleucina 8 (IL-8).

LPS: lipopolisacárido. 
reducción significativa del TGF- $\beta$ en un $61,6 \pm$ $20,7 \%$, en comparación con el control + LPS, resultando un descenso significativo $(p<0,0001)$ (fig. 10).

En resumen, el tratamiento con LPS en dosis de $100 \mathrm{ng} / \mathrm{ml}$ indujo significativamente la producción de TNF- $\alpha$, IL-1 $\alpha$, IL-6 e IL-8, mostrando que el LPS indujo la activación de la respuesta inflamatoria por estas vías. Cuando las células del folículo piloso se incubaron con MSM en dosis de $0,05 \mathrm{mg} / \mathrm{ml}$, los resultados mostraron que el tratamiento redujo significativamente la expresión de TNF- $\alpha$, IL- 6 , IL- 8 y TGF- $\beta$, a razón de un $49,9 \pm 29,1 \%$, un $73 \pm 29,9 \%$, un $63,1 \pm 30,4 \%$ y un $61,6 \pm 20,7 \%$, respectivamente, en comparación con el control + LPS.

Con los datos observados, se podría plantear el uso de MSM en el tratamiento de la AAF, en busca de sus efectos beneficiosos antiinflamatorios, al tratar el factor inflamatorio identificado como uno de los factores implicados en la AAF, responsable de originar cambios tisulares y producir fibrosis perifolicular en la alopecia androgénica y microinflamación en el tercio superior del folículo, que contribuyen al proceso de miniaturización del cabello y a la apoptosis celular observada en la AAF.

\section{Efecto sobre la expresión del indicador antioxidante glutatión}

A la hora de medir el estrés oxidativo a nivel celular, el principal indicador es el GSH, siendo el antioxidante natural por excelencia. Otra forma habitual de medir el estado oxidativo de la célula es evaluar el cociente GSH/GSSG (cociente entre la forma reducida y la forma oxidada del GSH), pues, a menor valor de este cociente, se considera que existe un peor estado oxidativo de la célula y mayor riesgo de toxicidad celular. Los resultados del estudio llevado a cabo en un cultivo celular de papila dérmica oxidada demostraron que el tratamiento con MSM en dosis de $0,5 \mathrm{mg} / \mathrm{ml}$ tuvo un efecto incremental significativo del GSH de un $41,8 \pm 10,5 \%$, y del $25,6 \pm 8,9 \%$ sobre el cociente GSH/GSSG, respecto al grupo de control sin MSM (fig. 11).

TGF- $\beta$ (LPS)

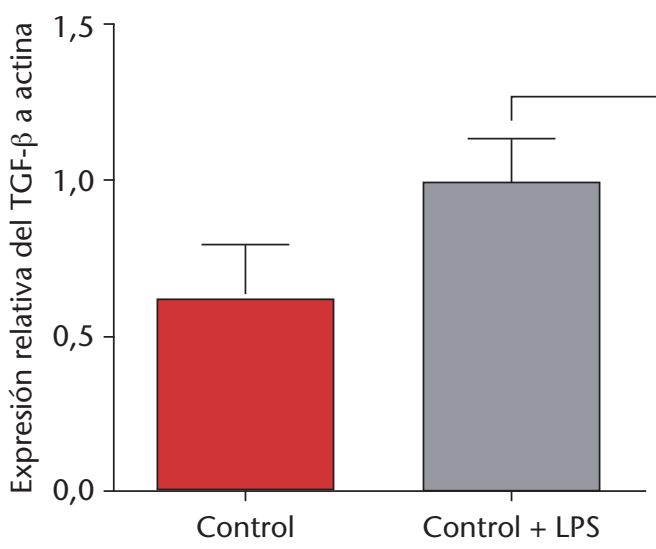

$61,6 \pm 20,7 \%$

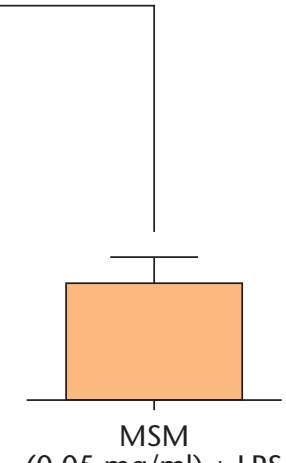

$(0,05 \mathrm{mg} / \mathrm{ml})+\mathrm{LPS}$

Figura 10. Efecto del metilsulfonilmetano (MSM) sobre la expresión del factor de crecimiento transformante beta (TGF- $\beta$ ). LPS: lipopolisacárido. 


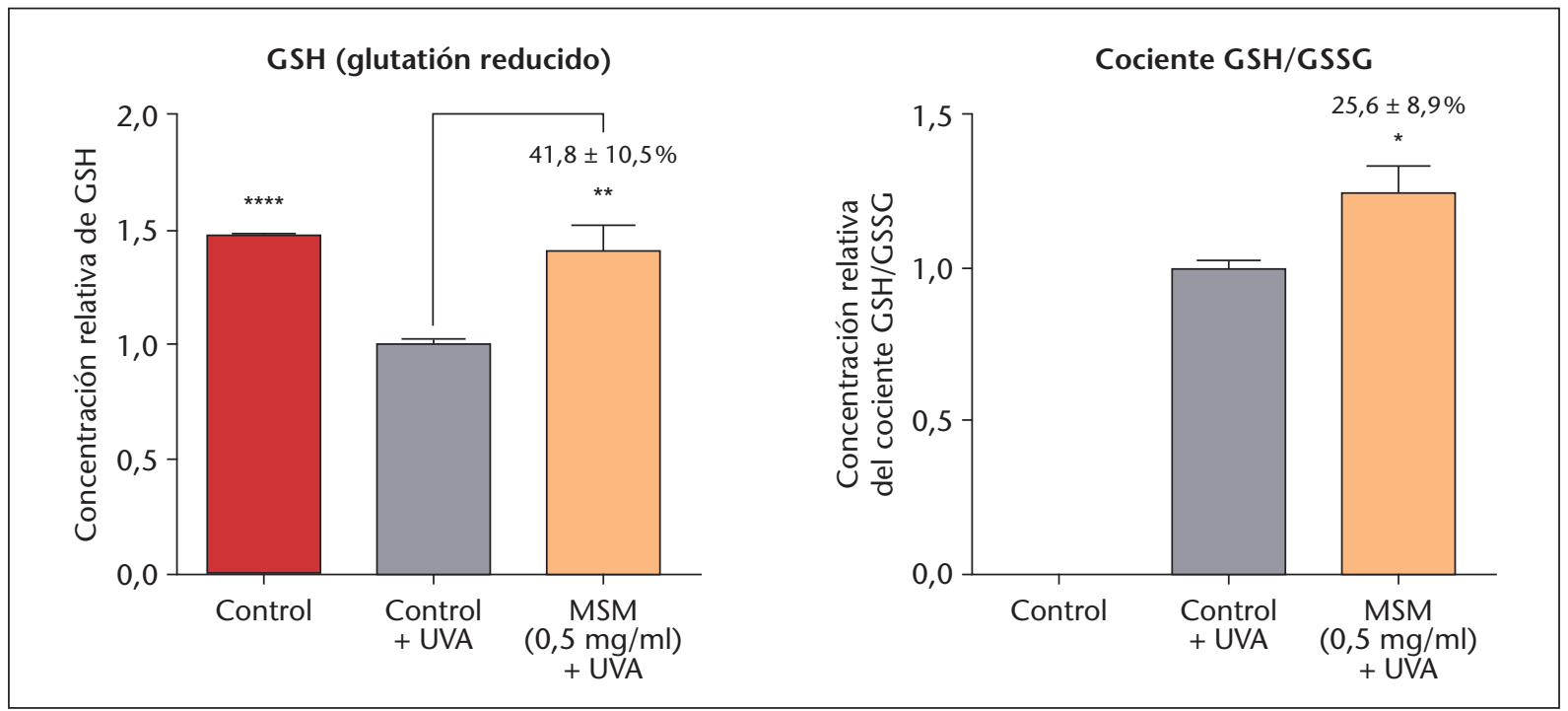

Figura 11. Efecto del metilsulfonilmetano (MSM) sobre la expresión del indicador antioxidante glutatión (GSH).

GSSG: glutatión oxidado; UVA: radiación ultravioleta A.

\section{CONCLUSIONES}

La AAF es la principal causa de pérdida de pelo en las mujeres adultas y conlleva un importante impacto psicológico y en la calidad de vida. Se produce por una miniaturización progresiva de los folículos pilosos, que conduce a una disminución difusa de la densidad del cabello en determinadas áreas del cuero cabelludo, con unas características clínicas y unos patrones histológicos definidos. La patogenia de la AAF responde a un mecanismo fisiopatológico multifactorial, en el que están implicados factores genéticos, hormonales, inflamatorios y oxidativos. Con el fin de conseguir un abordaje clínico lo más integral posible de la AAF, los nuevos tratamientos para esta enfermedad deberían actuar sobre todos los factores identificados hasta el momento implicados en la AAF.

A lo largo de este artículo, hemos analizado las tres dianas principales de tratamiento para cada uno de estos factores: el factor hormonal y la inhibición de la enzima $5 \alpha$-reductasa, el factor oxidativo y la búsqueda de equilibrio a favor de factores antioxidantes, y el factor inflamatorio y la búsqueda de efectos antiinflamatorios.

$\mathrm{Al}$ mismo tiempo y para cada una de estas dianas, hemos querido plantear una estrategia para alcanzar los objetivos planteados: incrementar la actividad inhibidora de la enzima $5 \alpha$-reductasa de los extractos que han mostrado el mejor nivel de eficacia y seguridad a lo largo de su experiencia clínica, e incorporar una nueva molécula que actúe de manera sinérgica con los extractos anteriores y aporte un valor añadido al tratamiento de la AAF por sus efectos antioxidantes y antiinflamatorios que vendría a proporcionar ese abordaje clínico más integral que nos proponíamos al principio de este artículo.

Como conclusiones de todo lo planteado y como propuestas de tendencias futuras en pro de una mejora en la efectividad del tratamiento de la AAF, se propone, una vez identificados los extractos Pygeum + Serenoa como el tratamiento que cuenta con el mejor aval de evidencia científica publicada, existiendo margen de eficacia y seguridad suficientes, y habiendo mostrado una relación directa entre dosis y respuesta inhibidora de la enzima $5 \alpha$-reductasa, un aumento de dosis con el fin de conseguir un incremento de su actividad inhibidora de la enzima $5 \alpha$-reductasa, manteniendo su buena tolerabilidad. Por otro lado, y a fin de sumar a lo anterior un efecto positivo sobre los factores oxidativo e inflamatorio, una 
vez identificada la molécula de MSM como fuente de azufre para la síntesis de queratina esencial en el ciclo de vida del cabello y como el agente que nos puede aportar ese efecto antioxidante y antiinflamatorio en el folículo piloso, se propone la incorporación de esta molécula en la dosis necesaria para obtener los efectos buscados en la composición de un preparado que contenga los extractos Pygeum + Serenoa.

La bibliografía nos describe que la dosis ideal de MSM se iniciaría con $1 \mathrm{~g}$ /día, pudiéndose incrementar hasta los 5-6 g/día divididos en dos dosis. Los estudios de toxicidad han demostrado que no se aprecian efectos adversos en animales, incluso, utilizando dosis hasta siete veces superiores a las máximas recomendadas en humanos. Por otra parte, el MSM es un producto reconocido por la Food and Drug Administration como GRAS (generally recognized as safe) en dosis que llegan hasta los $5 \mathrm{~g} /$ día.

Teniendo en cuenta los planteamientos desarrollados a lo largo de este artículo, las nuevas tendencias terapéuticas para el tratamiento de la mujer con AAF, además de desarrollar su actividad a nivel enzimático con un aumento de su capacidad inhibitoria de la encima $5 \alpha$ reductasa, podrían actuar reduciendo el estrés oxidativo y la microinflamación, con el fin de regularizar el metabolismo del folículo piloso e impedir la apoptosis de sus células, atacando los tres factores fisiopatológicos desencadenantes de la AAF.

En resumen, las nuevas tendencias de tratamiento en la AAF idealmente podrían contemplar:

- Aumento de la actividad inhibidora de la $5 \alpha$ reductasa.

- Actividad antioxidante para reducir el estrés oxidativo.

- Actividad antiinflamatoria para reducir la microinflamación.

- Aumento del aporte de azufre más asimilable, para la síntesis de queratina.

Todo ello debería redundar en un abordaje clínico más integral de la AAF, en el que se consi- guiera no solo frenar la caída del cabello, sino también una revitalización del folículo piloso y un alargamiento de la fase anágena. En definitiva, actuar sobre cada uno de los factores fisiopatológicos identificados actualmente en la AAF, para mejorar la calidad del pelo afectado y la calidad de vida de la mujer afectada por esta patología.

\section{BIBLIOGRAFÍA}

1. Guerra A, González-Guerra E, Borrás JM. Alopecia androgénica femenina: nuevos factores fisiopatológicos y futuras tendencias para un abordaje clínico más integral. Más Dermatol. 2016;(26):25-36.

2. Will T, Ishani A, Stark G, MacDonald R, Mulrow C, Lau J. Serenoa repens for benign prostatic hyperplasia. Cochrane Database Syst Rev. 2000;(2):CD001423.

3. Wilt T, Ishani A, Mac Donald R, Rutks I, Stark G. Pygeum africanum for benign prostatic hyperplasia. Cochrane Database Syst Rev. 2002;(1):CD001044.

4. Brosnan JT, Brosnan ME. The sulfur-containing amino acids: an overview. J Nutr. 2006;136(6 Suppl):1636S-1640S.

5. Andriole GL, Kirby R. Safety and tolerability of the dual 5alphareductase inhibitor dutasteride in the treatment of benign prostatic hyperplasia. Eur Urol. 2003;44(1):82-8.

6. Guerra Tapia A. Androgenización y su tratamiento: el acetato de ciproterona 30 años después. Piel. 1999;14(10):532-8.

7. Wilt TJ, Ishani A, Rutks I, MacDonald R. Phytotherapy for benign prostatic hyperplasia. Public Health Nutr. 2000;3(4A): 459-72.

8. Borrás JM, Piqué N, Nieto C, González J. Efficacy and safety of a dietary supplement containing a lipid co-extract from Serenoa repens and Pygeum africanum for the treatment of androgenetic alopecia (AGA) in women. Results of a randomized, double-blind, placebo-controlled clinical trial. Más Dermatol. 2016;(25):5-14.

9. World Health Organization (WHO). WHO monographs on selected medicinal plants. Volume 2. Ginebra: WHO; 2002. Disponible en: http://apps.who.int/iris/bitstream/10665/ 42052/2/9241545372.pdf

10. Joung YH, Lim EJ, Darvin P, Chung SC, Jang JW, Do Park K, et al. MSM enhances $\mathrm{GH}$ signaling via the Jak2/STAT5b pathway in osteoblast-like cells and osteoblast differentiation through the activation of STAT5b in MSCs. PLoS ONE. 2012; 7(10):e47477.

11. Ameye LG, Chee WS. Osteoarthritis and nutrition. From nutraceuticals to functional foods: a systematic review of the scientific evidence. Arthritis Res Ther. 2006;8(4):R127.

12. Di Chiacchio N, Suárez Restrepo MV. Efficacy and safety of a topical formulation in patients with brittle nail syndrome: a randomized, single-blind, crossover, controlled study. Surg Cosmet Dermatol. 2015;7(1):26-32.

13. Nakhostin-Roohi B, Barmaki S, Khoshkhahesh F, Bohlooli S. Effect of chronic supplementation with methylsulfonylmethane on oxidative stress following acute exercise in untrained healthy men. J Pharm Pharmacol. 2011;63(10):1290-4. 